\title{
Morphological and phylogenetic studies of Pleopunctum gen. nov. (Phaeoseptaceae, Pleosporales) from China
}

\author{
Liu NG ${ }^{1,2,3,4,5}$, Hyde $\mathrm{KD}^{4,5}$, Bhat $\mathrm{DJ}^{6}$, Jumpathong $\mathrm{J}^{3}$ and Liu $\mathrm{JK}^{1^{*, 2}}$ \\ ${ }^{1}$ School of Life Science and Technology, University of Electronic Science and Technology of China, Chengdu 611731, \\ P.R. China \\ ${ }^{2}$ Guizhou Key Laboratory of Agricultural Biotechnology, Guizhou Academy of Agricultural Sciences, Guiyang 550006, \\ P.R. China \\ ${ }^{3}$ Faculty of Agriculture, Natural Resources and Environment, Naresuan University, Phitsanulok 65000, Thailand \\ ${ }^{4}$ Center of Excellence in Fungal Research, Mae Fah Luang University, Chiang Rai 57100, Thailand \\ ${ }^{5}$ Mushroom Research Foundation, Chiang Rai 57100, Thailand \\ ${ }^{6}$ No. 128/1-J, Azad Housing Society, Curca, P.O., Goa Velha 403108, India
}

Liu NG, Hyde KD, Bhat DJ, Jumpathong J, Liu JK 2019 - Morphological and phylogenetic studies of Pleopunctum gen. nov. (Phaeoseptaceae, Pleosporales) from China. Mycosphere 10(1), 757-775, Doi 10.5943/mycosphere/10/1/17

\begin{abstract}
A new hyphomycete genus, Pleopunctum, is introduced to accommodate two new species, $P$. ellipsoideum sp. nov. (type species) and $P$. pseudoellipsoideum sp. nov., collected from decaying wood in Guizhou Province, China. The genus is characterized by macronematous, mononematous conidiophores, monoblastic conidiogenous cells and muriform, oval to ellipsoidal conidia often with a hyaline, elliptical to globose basal cell. Phylogenetic analyses of combined LSU, SSU, ITS and TEF1 $\alpha$ sequence data of 55 taxa were carried out to infer their phylogenetic relationships. The new taxa formed a well-supported subclade in the family Phaeoseptaceae and basal to Lignosphaeria and Thyridaria macrostomoides. Divergence time estimation based on LSU, SSU and TEF1 $\alpha$ sequence data was performed to provide additional evidence for the establishment of Phaeoseptaceae, which diverged approximately 88 MYA.
\end{abstract}

Key words - 3 new taxa - Asexual morph - Divergence times - Dothideomycetes - Multi-genes Taxonomy

\section{Introduction}

Hyphomycetes are the asexually reproducing part of the life cycle of ascomycetes and basidiomycetes (Seifert et al. 2011). They are a diverse group belonging to different classes, families and genera as shown by modern molecular phylogenetic analyses (Jeewon et al. 2003, Hyde et al. 2013, Maharachchikumbura et al. 2016, Dai et al. 2017, Doilom et al. 2017, Wijayawardene et al. 2017, Lu et al. 2018). Colonies of dematiaceous hyphomycetes on natural substrates are normally effuse, conspicuous, velvety and brown or black (Ellis 1971, 1976, Bhat 2010, Seifert et al. 2011, Luo et al. 2017). Punctiform colonies are common in dematiaceous hyphomycetes. Although punctiform colonies on natural substrates are similar when observed under the stereoscope, the morphology of conidia is quite diverse, and their phylogenetic affinities are rather intriguing. Historically, it has been uncommon to link hyphomycetes with their sexual morphs, since many asexual morphs have permanently lost the potential to produce a sexual morph (Seifert et al. 2011). 
However, with the advent of DNA based methods, taxonomic placement of many hyphomycetes with or without sexual morphs has been possible (Tsui et al. 2006, Shenoy et al. 2007, Wang et al. 2007). This has shown that many morphologically similar hyphomycetous groups are polyphyletic (Shenoy et al. 2006, Pinnoi et al. 2007, Klaubauf et al. 2014, Lu et al. 2018).

The cheirosporous genus Dictyosporium Corda and their relatives are placed in Dictyosporiaceae (Boonmee et al. 2016, Yang et al. 2018). Both Hermatomyces Speg. and Lentimurispora N.G. Liu, Bhat \& K.D. Hyde can produce cushion-like, lenticular conidia with dark brown central cells and subhyaline to pale brown peripheral cells. However, Hermatomyces belongs to Hermatomycetaceae (Hashimoto et al. 2017), while Lentimurispora is accommodated in Lentimurisporaceae (Liu et al. 2018). All of the families, Dictyosporiaceae, Hermatomycetaceae and Lentimurisporaceae belong to the Dothideomycetes. Yang et al. (2016) established the order Fuscosporellales in the subclass Hypocreomycetidae (Sordariomycetes) based on fungi isolated from freshwater. Two genera, Fuscosporella Jing Yang, Bhat \& K.D. Hyde and Parafuscosporella Jing Yang, Bhat \& K.D. Hyde form black, punctiform colonies with obpyriform conidia on submerged twigs. Canalisporium Nawawi \& Kuthub. also resides in the subclass Hypocreomycetidae (Sordariomycetes). Unlike conidia of Fuscosporella and Parafuscosporella having a basal septum, Canalisporium produces muriform conidia (Goh et al. 1998, Sri-Indrasutdhi et al. 2010). Besides two largest classes of Ascomycota (Dothideomycetes and Sordariomycetes), punctiform colonies have also been reported from other classes. Aculeata W. Dong, H. Zhang \& K.D. Hyde was recently introduced by Dong et al. (2018) in Herpotrichiellaceae (Eurotiomycetes). Conidia of Aculeata are olive to brown, vesiculate, cruciately septate, bearing densely packed, subulate, obtuse, brown to black spines (Dong et al. 2018). There are also some hyphomycetous genera treated as incertae sedis due to lack of molecular data. For example, Vanakripa Bhat, W.B. Kendr. \& Nag Raj was introduced by Bhat \& Kendrick (1993) with the type species V. gigaspora Bhat, Kendrick \& Nag Raj. Since then, eight species were described in Vanakripa but none of them have DNA sequence data in public repositories (Tsui et al. 2003, Hu et al. 2010, Leão-Ferreira et al. 2013). This genus is now assigned as ascomycetes incertae sedis. The conidial morphology in Vanakripa is similar to those of Fuscosporella and Parafuscosporella, however, their conidiogenous cells are distinguishable.

While investigating dematiaceous hyphomycetes in China and Thailand, two interesting hyphomycetes with punctiform colonies were collected from decaying wood in China. Phylogenetic analyses based on the combined LSU, SSU, ITS and TEF1 $\alpha$ sequence data indicated that these two taxa represent a new genus in Phaeoseptaceae (Pleosporales). Therefore, Pleopunctum gen. nov., is introduced to accommodate $P$. ellipsoideum and $P$. pseudoellipsoideum spp. nov. with detailed morphological studies and supported by multi-gene phylogenetic analyses. In addition, we also estimated the divergence time to further support the establishment of Phaeoseptaceae.

\section{Materials \& Methods}

\section{Collections and examination of specimens}

Fresh samples of decaying wood were collected from Guizhou Province, China. The samples were processed and examined following the method described in Taylor \& Hyde (2003). The samples were incubated in plastic boxes with sterile and moist tissue at $25-30{ }^{\circ} \mathrm{C}$ for 3 days, and then examined using a Motic SMZ 168 Series dissecting microscope. Fruiting bodies of the new taxa were mounted in a drop of water for microscopic studies and photomicrography. The species were examined with a Nikon ECLIPSE 80i compound microscope fitted with a Canon 600D digital camera. Measurements were performed using the Tarosoft (R) Image Frame Work software (Liu et al. 2010) and photo-plates were prepared using Adobe Photoshop CS3 software (Adobe Systems, USA).

Single conidium isolations were carried out following the method described in Chomnunti et al. (2014). Germinated conidia were individually transferred to potato dextrose agar (PDA) media plates and incubated at $25{ }^{\circ} \mathrm{C}$. Dried specimens were deposited in the herbarium of Mae Fah Luang 
University (MFLU), Chiang Rai, Thailand. Pure cultures were deposited in the Mae Fah Luang University Culture Collection (MFLUCC). Facesoffungi (FoF) numbers were acquired as in Jayasiri et al. (2015) and Index Fungorum numbers as in Index Fungorum (2019).

\section{DNA extraction, PCR amplification and sequencing}

A sterile scalpel was used to scrape fresh mycelia from pure cultures growing on PDA medium for one month at $25{ }^{\circ} \mathrm{C}$. Genomic DNA was extracted using DNA Extraction Kit (Sangon Biotech, Shanghai, P.R. China) following the manufacture's protocol. Four different gene regions, the nuclear large subunit rDNA (28S, LSU), the nuclear small subunit rDNA (18S, SSU), internal transcribed spacer (ITS) and the translation elongation factor 1-alpha gene (TEF1 $\alpha$ ) were selected for study. Part of LSU locus was amplified with the primers LR0R and LR5 (Vilgalys \& Hester 1990), part of SSU with primers NS1 and NS4 (White et al. 1990), part of ITS with primers ITS5 and ITS4 (White et al. 1990), and part of TEF1 $\alpha$ with primers 983F and 2218R (Rehner \& Buckley 2005). Polymerase chain reaction (PCR) was carried out in $25 \mu 1$ reaction volume containing 12.5

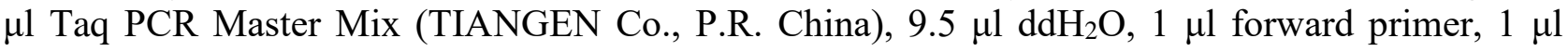
reverse primer and $1 \mu \mathrm{LNA}$ template. PCR conditions for LSU, SSU, ITS and TEF1 $\alpha$ were as follows: $3 \mathrm{~min}$ at $94{ }^{\circ} \mathrm{C}$ (initial denaturation), followed by 40 cycles of $45 \mathrm{~s}$ at $94{ }^{\circ} \mathrm{C}$ (denaturation), $50 \mathrm{~s}$ at $56{ }^{\circ} \mathrm{C}$ (annealing), $1 \mathrm{~min}$ at $72{ }^{\circ} \mathrm{C}$ (extension), with a final extension of $10 \mathrm{~min}$ at $72{ }^{\circ} \mathrm{C}$. Purified PCR products were sequenced by Sangon Biotech (Shanghai, P.R. China).

\section{Phylogenetic analyses}

Fifty-five strains representing Amorosiaceae, Halotthiaceae, Lentimurisporaceae, Lophiostomaceae, Phaeoseptaceae, Sporormiaceae and Teichosporaceae, along with the outgroup Lindgomyces ingoldianus KH100 (Table 1) in the order Pleosporales used for this study were obtained from GenBank baed on blast search results and other published studies (Mantle et al. 2006, Kruys \& Wedin 2009, Mugambi \& Huhndorf 2009, Zhang et al. 2013, Thambugala et al. 2015, Phukhamsakda et al. 2016a, Hyde et al. 2018, Liu et al. 2017, 2018). The multiple alignments were automatically performed by online MAFFT version 7 (Katoh \& Standley 2013) and BioEdit (Hall 1999). Four genes were combined using BioEdit. Alignments were checked visually and optimized manually using AliView (Larsson 2014) where necessary. The final alignment was deposited in TreeBASE (submission ID: 24638). Sequences derived in this study were deposited in GenBank (Table 1).

Maximum likelihood analysis was performed using RAxML (Stamatakis 2006). The tree search included 1,000 non-parametric bootstrap replicates and the best scoring tree was selected from suboptimal trees under the GTRGAMMA substitution model. The resulting replicates were plotted on to the best scoring tree obtained previously.

Maximum parsimony analysis was performed with the heuristic search in PAUP v. 4.0b10 (Swofford 2002). Gaps in the alignment were treated as missing characters and all characters were unordered. Maxtrees were unlimited. Branches of zero length were collapsed and all multiple, equally parsimonious trees were saved. Clade stability was assessed using a bootstrap (BT) analysis with 1,000 replicates, each with 10 replicates of random stepwise addition of taxa (Hillis \& Bull 1993).

Bayesian analyses were performed in MrBayes 3.2.6 (Ronquist et al. 2012). The program MrModeltest 2.2 (Nylander 2004) was used to determine the best nucleotide substitution model for each data partition. GTR $+\mathrm{I}+\mathrm{G}$ substitution model with gamma rates and dirichlet base frequencies was decided for LSU, SSU, ITS and TEF1 $\alpha$ sequences. The Markov Chain Monte Carlo (MCMC) sampling approach was used to calculate posterior probabilities (PP) (Rannala \& Yang 1996). Bayesian analyses of four simultaneous Markov chains were run for 5,000,000 generations with trees sampled every 1,000th generations. The first $20 \%$ of trees, representing the burn-in phase of the analyses, were discarded and the remaining trees were used for calculating posterior probabilities (PP) in the majority rule consensus tree. 


\section{Fossil calibration, divergence time and evolutionary rate estimations}

The fossil calibrations used in the analyses followed the methodology described in Phukhamsakda et al. (2016b). The related sequences in the class Dothideomycetes are listed in Table 2. Reliable fossils and one secondary calibration were selected for the divergence times estimations based on the phylogenetic analyses. The fossil Metacapnodium succinum (Metacapnodiaceae) was used to calibrate the minimum age of Capnodiales (normal distribution, mean $=100, \mathrm{SD}=150$, providing 95\% credibility interval of 346 MYA) (Rikkinen et al. 2003, Hongsanan et al. 2016, Pérez-Ortega et al. 2016, Phukhamsakda et al. 2016b, Samarakoon et al. 2019). The fossil Margaretbarromyces dictyosporus was used to calibrate the crown age of Aigialus (Aigialaceae) (gamma distribution, offset $=35$, shape $=1.0$, scale $=25$, providing 95\% credibility interval of 110 MYA) (Mindell et al. 2007, Phukhamsakda et al. 2016b). The split between Arthoniomycetes and Dothideomycetes was calibrated using the results from Phukhamsakda et al. (2016b) as the secondary calibration (normal distribution, mean $=300$, SD = 50, providing 95\% credibility interval of 382 MYA).

Evolutionary estimation was performed by BEAST 1.8.0 (Drummond et al. 2012). Aligned sequence data were partitioned separately for each LSU, SSU, TEF1 $\alpha$ data set, and were loaded to prepare an XML file constructed with BEAUti v1.8.0. Clock and substitution models were set to be unlinked (independently estimated foreach gene partition), while the tree prior parameters were set to be linked across partitions (concatenation). We applied a lognormal relaxed clock (uncorrelated). The tree prior was shared by all tree models; this consisted of a birth/death in complete sampling tree prior and was used to model the speciation of nodes in the topology with uniform prior on probability of splits and extinctions. The analysis was performed for 100 million generations in BEAST v1.8.0, and sampling parameters every 1,000 generations. Tracer v1.6 (Rambaut et al. 2014) was used to check the effective sample sizes (ESS) (ESS $>200$ ). The first of $20 \%$ trees were discarded as a burn-in phase. The remaining trees were combined in LogCombiner v.1.8.0. A maximum clade creditability tree was generated by Tree Annotator v1.8.0.

Trees were visualized with FigTree v1.4.0 (Rambaut 2006) and the layout was edited using Adobe Illustrator CS6 software (Adobe Systems, USA).

Table 1 Taxa used in in this study. The new taxa are indicated in bold.

\begin{tabular}{|c|c|c|c|c|c|}
\hline Species & Strain numbers & LSU & SSU & ITS & TEF1a \\
\hline Alpestrisphaeria terricola & SC-12 & JX985750 & JX985749 & JN662930 & \\
\hline Amorosia littoralis & NN 6654 & AM292055 & AM292056 & AM292047 & \\
\hline Angustimassarina populi & MFLUCC 13-0034 & KP888642 & KP899128 & KP899137 & KR075164 \\
\hline Bahusandhika indica & GUFCC 18001 & KF460274 & & KF460273 & \\
\hline $\begin{array}{l}\text { Berkleasmium } \\
\text { micronesicum }\end{array}$ & BCC 8141 & DQ280272 & DQ280268 & DQ280262 & \\
\hline $\begin{array}{l}\text { Berkleasmium } \\
\text { nigroapicale }\end{array}$ & BCC 8220 & DQ280273 & DQ280269 & DQ280261 & \\
\hline $\begin{array}{l}\text { Biappendiculispora } \\
\text { japonica }\end{array}$ & MAFF 239452 & AB619005 & AB618686 & LC001728 & LC001744 \\
\hline $\begin{array}{l}\text { Brunneoclavispora } \\
\text { bambusae }\end{array}$ & MFLUCC 11-0177 & KT426562 & & MG520957 & \\
\hline $\begin{array}{l}\text { Capulatispora } \\
\text { sagittiformis }\end{array}$ & JCM 15100 & AB369267 & AB618693 & AB369268 & LC001756 \\
\hline $\begin{array}{l}\text { Coelodictyosporium } \\
\text { pseudodictyosporium }\end{array}$ & MFLUCC 13-0451 & KR025862 & & KR025858 & \\
\hline Decaisnella formosa & BCC 25617 & GQ925847 & GQ925834 & & GU479850 \\
\hline Decaisnella formosa & BCC 25616 & GQ925846 & GQ925833 & & GU479851 \\
\hline Exosporium stylobatum & CBS 160.30 & JQ044447 & & JQ044428 & \\
\hline Forliomyces uniseptata & MFLUCC 15-0765 & KU721762 & KU721767 & KU721772 & \\
\hline
\end{tabular}


Table 1 Continued.

\begin{tabular}{|c|c|c|c|c|c|}
\hline Species & Strain numbers & LSU & SSU & ITS & TEF1a \\
\hline Guttulispora crataegi & MFLUCC 13-0442 & KP888639 & KP899125 & KP899134 & KR075161 \\
\hline Halotthia posidoniae & BBH 22481 & GU479786 & GU479752 & & \\
\hline $\begin{array}{l}\text { Lentimurispora } \\
\text { urniformis }\end{array}$ & MFLUCC 18-0497 & MH179144 & MH179160 & & MH188055 \\
\hline Lignosphaeria fusispora & MFLUCC 11-0377 & KP888646 & & KР899140 & \\
\hline $\begin{array}{l}\text { Lignosphaeria } \\
\text { thailandica }\end{array}$ & MFLUCC 11-0376 & KP888645 & & KP899139 & \\
\hline Lindgomyces ingoldianus & KH 100 & AB521737 & AB521720 & & \\
\hline $\begin{array}{l}\text { Lophiohelichrysum } \\
\text { helichrysi }\end{array}$ & MFLUCC 15-0701 & КТ333436 & КТ333437 & KT333435 & KT427535 \\
\hline $\begin{array}{l}\text { Lophiopoacea } \\
\text { paramacrostoma }\end{array}$ & MFLUCC 11-0463 & KP888636 & KP899122 & & \\
\hline $\begin{array}{l}\text { Lophiostoma } \\
\text { macrostomum }\end{array}$ & JCM 13544 & AB619010 & AB618691 & JN942961 & LC001751 \\
\hline Massarina corticola & CBS 154.93 & FJ795448 & FJ795491 & & \\
\hline Mauritiana rhizophorae & BCC 28866 & GU371824 & GU371832 & & GU371817 \\
\hline $\begin{array}{l}\text { Neolophiostoma } \\
\text { pigmentatum }\end{array}$ & MFLUCC 10-0129 & КT324588 & КТ324589 & КT324587 & KT324590 \\
\hline $\begin{array}{l}\text { Neotrematosphaeria } \\
\text { biappendiculata }\end{array}$ & KTC 1124 & GU205227 & GU205256 & & \\
\hline Paucispora quadrispora & КH 448 & LC001722 & LC001720 & LC001733 & LC001754 \\
\hline Phaeoseptum aquaticum & CBS 123113 & JN644072 & & & \\
\hline Phaeoseptum terricola & MFLUCC 10-0102 & MH105779 & MH105780 & MH105778 & MH105781 \\
\hline $\begin{array}{l}\text { Platystomum } \\
\text { compressum }\end{array}$ & MFLUCC 13-0343 & KP888643 & KР899129 & & KR075165 \\
\hline $\begin{array}{l}\text { Pleopunctum } \\
\text { ellipsoideum }\end{array}$ & MFLUCC 19-0390 & MK804517 & MK804514 & MK804512 & MK828510 \\
\hline $\begin{array}{l}\text { Pleopunctum } \\
\text { pseudoellipsoideum }\end{array}$ & MFLUCC 19-0391 & MK804518 & & MK804513 & MK828511 \\
\hline Preussia funiculata & CBS 659.74 & GU301864 & GU296187 & & GU349032 \\
\hline $\begin{array}{l}\text { Pseudolophiostoma } \\
\text { vitigenum }\end{array}$ & JCM 13534 & AB619015 & AB618697 & LC001735 & LC001761 \\
\hline $\begin{array}{l}\text { Pseudoplatystomum } \\
\text { scabridisporum }\end{array}$ & BCC 22835 & GQ925844 & GQ925831 & & GU479857 \\
\hline Sigarispora ravennica & MFLUCC 14-0005 & KP698414 & KP698415 & KР698413 & \\
\hline Sparticola junci & MFLUCC 15-0030 & KU721765 & KU721770 & KU721775 & KU727898 \\
\hline Sporormia fimetaria & $\begin{array}{l}\text { UPS:Dissing } \\
\text { Gr.81.194 }\end{array}$ & GQ203729 & & GQ203769 & \\
\hline Sporormiella minima & CBS 52450 & DQ468046 & & DQ468026 & \\
\hline $\begin{array}{l}\text { Sulcosporium } \\
\text { thailandicum }\end{array}$ & MFLUCC 12-0004 & KT426563 & KT426564 & MG520958 & \\
\hline $\begin{array}{l}\text { Teichospora } \\
\text { aurantiacinotata }\end{array}$ & GKM 1238 & GU385173 & & & \\
\hline $\begin{array}{l}\text { Teichospora } \\
\text { austroafricana }\end{array}$ & CBS 122674 & EU552116 & & EU552116 & \\
\hline Teichospora cruciformis & SMH 5151 & GU385211 & & & \\
\hline Teichospora kenyensis & GKML $100 \mathrm{Na}$ & GU385189 & & & GU327766 \\
\hline Teichospora parva & GKM 169N & GU385165 & & & GU327768 \\
\hline Teichospora striata & JK 5678I & GU301813 & GU296149 & & GU479852 \\
\hline Teichospora tennesseensis & ANM 911 & GU385207 & & & GU327769 \\
\hline Teichospora thailandica & MFLUCC 13-0284 & KР888647 & KР899131 & КР899141 & KR075167 \\
\hline
\end{tabular}


Table 1 Continued.

\begin{tabular}{llllll}
\hline Species & Strain numbers & LSU & SSU & ITS & TEF1 $\boldsymbol{\alpha}$ \\
\hline $\begin{array}{l}\text { Teichospora uniseriata } \\
\begin{array}{l}\text { Thyridaria } \\
\text { macrostomoides }\end{array}\end{array}$ & ANM 909 & GU385206 & & & \\
$\begin{array}{l}\text { Thyridaria } \\
\text { macrostomoides }\end{array}$ & GKM 1033 & GU385190 & & & GU327776 \\
$\begin{array}{l}\text { Thyridaria } \\
\text { macrostomoides }\end{array}$ & GKM 224N & GU385185 & & & GU327778 \\
$\begin{array}{l}\text { Vaginatispora aquatica } \\
\text { Westerdykella ornata }\end{array}$ & MFLUCC 11-0083 & KJ591576 & KJ591575 & KJ591577 & \\
\hline
\end{tabular}

Table 2 Taxa used for divergence time estimate in this study.

\begin{tabular}{|c|c|c|c|c|}
\hline Species & Strain & LSU & SSU & TEF1a \\
\hline Aigialus grandis & BCC 18419 & GU479774 & GU479738 & GU479838 \\
\hline Aigialus mangrovei & BCC 33563 & GU479776 & GU479741 & GU479840 \\
\hline Aigialus parvus & BCC 18403 & GU479778 & GU479744 & GU479842 \\
\hline Aigialus rhizophorae & BCC 33572 & GU479780 & GU479745 & GU479844 \\
\hline Aliquandostipite khaoyaiensis & CBS 118232 & GU301796 & & GU349048 \\
\hline Amniculicola immersa & CBS 123083 & FJ795498 & GU456295 & GU456273 \\
\hline Amorosia littoralis & NN 6654 & AM292055 & AM292056 & \\
\hline Angustimassarina populi & MFLUCC 13-0034 & KР888642 & KР899128 & KR075164 \\
\hline Anteaglonium abbreviatum & GKM 219N & GQ221881 & & GQ221916 \\
\hline Anteaglonium parvulum & GKM 1029 & GQ221878 & & GQ221915 \\
\hline Arthonia dispersa & UPSC 2583 & AY571381 & AY571379 & \\
\hline Ascochyta pisi & AFTOL-ID 1583 & DQ678070 & DQ678018 & DQ677913 \\
\hline Ascocratera manglicola & BCC 09270 & GU479782 & GU479747 & GU479846 \\
\hline Astrosphaeriella fusispora & MFLUCC 10-0555 & КT955462 & КT955443 & KT955425 \\
\hline Bahusandhika indica & GUFCC 18001 & KF460274 & & \\
\hline Berkleasmium micronesicum & BCC 8141 & DQ280272 & DQ280268 & \\
\hline Berkleasmium nigroapicale & BCC 8220 & DQ280273 & DQ280269 & \\
\hline Bimuria novae-zelandiae & CBS 107.79 & NG_058623 & NG_061017 & \\
\hline Boeremia exigua & CBS 431.74 & JX681074 & EU754084 & KY484687 \\
\hline Botryosphaeria dothidea & CBS 115476 & NG_027577 & DQ677998 & DQ767637 \\
\hline Capnodium salicinum & CBS 131.34 & DQ678050 & DQ677997 & \\
\hline Caryospora minima & & EU196550 & EU196551 & \\
\hline Cladosporium cladosporioides & CBS 170.54 & AY213694 & DQ678004 & \\
\hline $\begin{array}{l}\text { Coelodictyosporium } \\
\text { pseudodictyosporium }\end{array}$ & MFLUCC 13-0451 & KR025862 & & \\
\hline Corynespora cassiicola & CBS 100822 & GU301808 & GU296144 & GU349052 \\
\hline Corynespora smithii & CABI 5649b & GU323201 & & GU349018 \\
\hline Cyclothyriella rubronotata & CBS 141486 & KX650544 & KX650507 & KX650519 \\
\hline Decaisnella formosa & BCC 25617 & GQ925847 & GQ925834 & GU479850 \\
\hline Decaisnella formosa & BCC 25616 & GQ925846 & GQ925833 & GU479851 \\
\hline Delitschia chaetomioides & SMH 3253.2 & GU390656 & & GU327753 \\
\hline Delitschia winteri & CBS 225.62 & DQ678077 & DQ678026 & DQ677922 \\
\hline Dendrographa leucophaea f. minor & & AF279382 & AF279381 & \\
\hline Dissoconium aciculare & CBS 204.89 & GU214419 & GU214523 & \\
\hline Exosporium stylobatum & CBS 160.30 & JQ044447 & & \\
\hline Gloniopsis calami & MFLUCC 15-0739 & KX646363 & KX669034 & KX671965 \\
\hline
\end{tabular}


Table 2 Continued.

\begin{tabular}{|c|c|c|c|c|}
\hline Species & Strain & LSU & SSU & TEF1a \\
\hline Gloniopsis praelonga & CBS 112415 & FJ161173 & FJ161134 & FJ161090 \\
\hline Guttulispora crataegi & MFLUCC 13-0442 & KP888639 & KP899125 & KR075161 \\
\hline Halotthia posidoniae & BBH 22481 & GU479786 & GU479752 & \\
\hline Herpotrichia diffusa & CBS 250.62 & DQ678071 & DQ678019 & DQ677915 \\
\hline Hypsostroma caimitalense & GKM 1165 & GU385180 & & \\
\hline Hypsostroma saxicola & SMH 5005 & GU385181 & & \\
\hline Hysterium angustatum & CBS 236.34 & FJ161180 & GU397359 & FJ161096 \\
\hline Jahnula seychellensis & SS 2113 & EF175665 & EF175644 & \\
\hline Katumotoa bambusicola & KT 1517a & AB524595 & AB524454 & AB539108 \\
\hline Lentimurispora urniformis & MFLUCC 18-0497 & Mh179144 & MH179160 & MH188055 \\
\hline Leptosphaeria doliolum & CBS 505.75 & GQ387576 & GQ387515 & GU349069 \\
\hline Leptoxyphium cacuminum & MFLUCC 10-0049 & JN832602 & JN832587 & \\
\hline Ligninsphaeria jonesii & MFLUCC 15-0641 & KU221037 & & \\
\hline Ligninsphaeria jonesii & GZCC 15-0080 & KU221038 & & \\
\hline Lignosphaeria fusispora & MFLUCC 11-0377 & KP888646 & & \\
\hline Lignosphaeria thailandica & MFLUCC 11-0376 & KP888645 & & \\
\hline Lindgomyces ingoldianus & ATCC 200398 & AB521736 & AB521719 & \\
\hline Lindgomyces rotundatus & KT 1096 & AB521740 & AB521723 & \\
\hline Lophiostoma macrostomum & KT 508 & AB619010 & AB618691 & LC001751 \\
\hline Lophiotrema lignicola & CBS 122364 & GU301836 & GU296166 & GU349072 \\
\hline Lophiotrema nucula & CBS 627.86 & GU301837 & GU296167 & GU349073 \\
\hline Massaria anomia & CBS 591.78 & GU301839 & GU296169 & \\
\hline Massaria inquinans & M 19 & HQ599402 & HQ599444 & HQ599342 \\
\hline Massarina corticola & CBS 154.93 & FJ795448 & FJ795491 & \\
\hline Massarina eburnea & CBS 473.64 & MH877786 & GU296170 & GU349040 \\
\hline Massariosphaeria phaeospora & CBS 611.86 & GU301843 & GU296173 & \\
\hline Mauritiana rhizophorae & BCC 28866 & GU371824 & GU371832 & GU371817 \\
\hline Melanomma pulvis-pyrius & CBS 124080 & GU456323 & GU456302 & GU456265 \\
\hline Murilentithecium clematidis & MFLUCC 14-0562 & KM408759 & NG_061185 & KM454445 \\
\hline Murispora rubicunda & IFRD 2017 & FJ795507 & GU456308 & GU456289 \\
\hline Neoastrosphaeriella krabiensis & MFLUCC 11-0025 & JN846729 & JN846739 & \\
\hline Neoroussoella bambusae & MFLUCC 11-0124 & KJ474839 & & KJ474848 \\
\hline $\begin{array}{l}\text { Neotrematosphaeria } \\
\text { biappendiculata }\end{array}$ & KTC 1124 & GU205227 & GU205256 & \\
\hline Nigrograna fuscidula & CBS 141476 & KX650547 & KX650509 & KX650522 \\
\hline Nigrograna mackinnonii & CBS 110022 & GQ387614 & GQ387553 & KF407985 \\
\hline Occultibambusa bambusae & MFLUCC 13-0855 & KU863112 & KU872116 & KU940193 \\
\hline Occultibambusa chiangraiensis & MFLUCC 16-0380 & KX655546 & KX655551 & KX655561 \\
\hline Ohleria modesta & MGC & KX650562 & & KX650533 \\
\hline Ohleria modesta & $\mathrm{OM}$ & KX650563 & KX650513 & KX650534 \\
\hline Paradictyoarthrinium diffractum & MFLUCC 13-0466 & KP744498 & KP753960 & \\
\hline Paradictyoarthrinium hydei & MFLUCC 17-2512 & MG747497 & MH454349 & \\
\hline Phaeoseptum aquaticum & CBS 123113 & JN644072 & & \\
\hline Phaeoseptum terricola & MFLUCC 10-0102 & MH105779 & MH105780 & MH105781 \\
\hline Phyllosticta capitalensis & CBS 226.77 & KF206289 & KF766300 & \\
\hline Piedraia hortae & CBS 480.64 & GU214466 & AY016349 & \\
\hline Pleomassaria siparia & CBS 279.74 & DQ678078 & DQ678027 & DQ677923 \\
\hline Pleopunctum ellipsoideum & MFLUCC 19-0390 & MK804517 & MK804514 & MK828510 \\
\hline
\end{tabular}


Table 2 Continued.

\begin{tabular}{|c|c|c|c|c|}
\hline Species & Strain & LSU & SSU & TEF1a \\
\hline Pleopunctum pseudoellipsoideum & MFLUCC 19-0391 & MK804518 & & MK828511 \\
\hline Pleospora herbarum & CBS 191.86 & MH873624 & GU238232 & KC584731 \\
\hline Preussia funiculata & CBS 659.74 & GU301864 & GU296187 & GU349032 \\
\hline Prosthemium orientale & MAFF 239509 & AB553748 & AB553641 & \\
\hline Pseudoastrosphaeriella bambusae & MFLUCC 11-0205 & KT955475 & & KT955437 \\
\hline $\begin{array}{l}\text { Pseudoastrosphaeriella } \\
\text { thailandensis }\end{array}$ & MFLUCC 10-0553 & КT955477 & KT955456 & KT955439 \\
\hline Psiloglonium araucanum & CBS 112412 & FJ161172 & FJ161133 & FJ161089 \\
\hline Pteridiospora javanica & MFLUCC 11-0159 & KJ742940 & KJ739607 & KJ739605 \\
\hline Racodium rupestre & L 346 & EU048583 & EU048575 & \\
\hline Racodium rupestre & L 424 & EU048582 & EU048577 & \\
\hline Rimora mangrovei & JK 5246A & GU301868 & GU296193 & \\
\hline Roccella fuciformis & Tehler 8171 & FJ638979 & & \\
\hline Roussoella nitidula & MFLUCC 11-0634 & KJ474842 & & KJ474851 \\
\hline Salsuginea ramicola & KT 2597.1 & GU479800 & GU479767 & GU479861 \\
\hline Salsuginea ramicola & KT 2597.2 & GU479801 & GU479768 & GU479862 \\
\hline Schismatomma decolorans & Ertz 5003 (BR) & NG_027622 & NG_013155 & \\
\hline Scorias spongiosa & CBS 325.33 & KF901821 & & \\
\hline Sigarispora ravennica & MFLUCC 14-0005 & КР698414 & KР698415 & \\
\hline Sporormia fimetaria & $\begin{array}{l}\text { UPS:Dissing } \\
\text { Gr.81.194 }\end{array}$ & GQ203729 & & \\
\hline Teichospora parva & GKM 169N & GU385165 & & GU327768 \\
\hline Teichospora striata & JK 5678I & GU301813 & GU296149 & GU479852 \\
\hline Teichospora tennesseensis & ANM 911 & GU385207 & & GU327769 \\
\hline Tetraplosphaeria sasicola & MAFF 239677 & AB524631 & AB524490 & \\
\hline Thyridaria acaciae & CBS 138873 & КР004497 & & \\
\hline Thyridaria broussonetiae & CBS 141481 & KX650568 & KX650515 & KX650539 \\
\hline Thyridaria macrostomoides & GKM 1033 & GU385190 & & GU327776 \\
\hline Thyridaria macrostomoides & GKM 1159 & GU385185 & & GU327778 \\
\hline Thyridaria macrostomoides & GKM 224N & GU385191 & & GU327777 \\
\hline Torula herbarum & CBS 111855 & KF443386 & KF443391 & KF443403 \\
\hline Torula hollandica & CBS 220.69 & KF443384 & KF443389 & KF443401 \\
\hline Triplosphaeria maxima & MAFF 239682 & AB524637 & AB524496 & \\
\hline Tubeufia chiangmaiensis & MFLUCC 11-0514 & KF301538 & KF301543 & KF301557 \\
\hline Tubeufia javanica & MFLUCC 12-0545 & KJ880036 & KJ880035 & KJ880037 \\
\hline Verruculina enalia & BCC 18401 & GU479802 & GU479770 & GU479863 \\
\hline Westerdykella ornata & CBS 379.55 & GU301880 & GU296208 & GU349021 \\
\hline Wicklowia aquatica & F 76-2 & GU045445 & GU266232 & \\
\hline Zopfia rhizophila & CBS 207.26 & DQ384104 & & \\
\hline
\end{tabular}

\section{Results}

\section{Phylogenetic analyses}

The manually adjusted LSU, SSU, ITS and TEF1 $\alpha$ alignment comprised a total of 3,622 characters (1,037 for LSU, 1,010 for SSU, 630 for ITS and 945 for TEF1 $\alpha$ ), including coded alignment gaps. Among them, 2,468 characters were constant, 353 variable characters were parsimony-uninformative, and number of parsimony-informative characters was 801 . Four hundred fifty-seven equally most parsimonious trees (Tree length $=3741, \mathrm{CI}=0.458, \mathrm{RI}=0.605, \mathrm{RC}=$ 
0.277, $\mathrm{HI}=0.542$ ) were yielded from the heuristic search. Maximum parsimony, maximum likelihood and Bayesian analyses of the combined dataset inferred similar topologies, respectively.

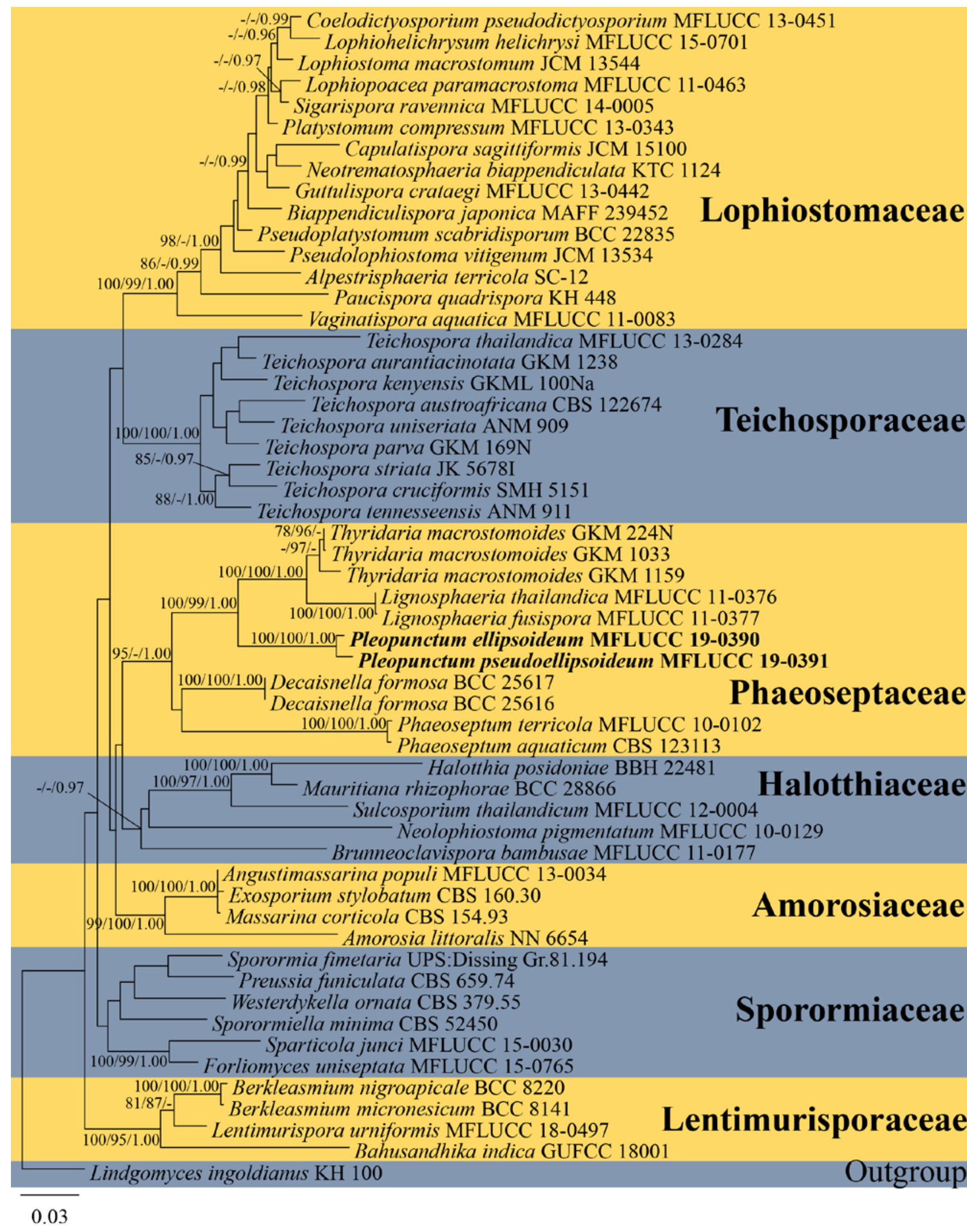

Figure 1 - Maximum likelihood (RAxML) tree based on analysis of a combined dataset of LSU, SSU, ITS and TEF1 $\alpha$ sequence data. Bootstrap support values for ML and MP greater than 75\% and Bayesian posterior probabilities above than 0.95 are given near nodes, respectively. The tree is rooted with Lindgomyces ingoldianus (KH 100). The new taxa are indicated in bold. 


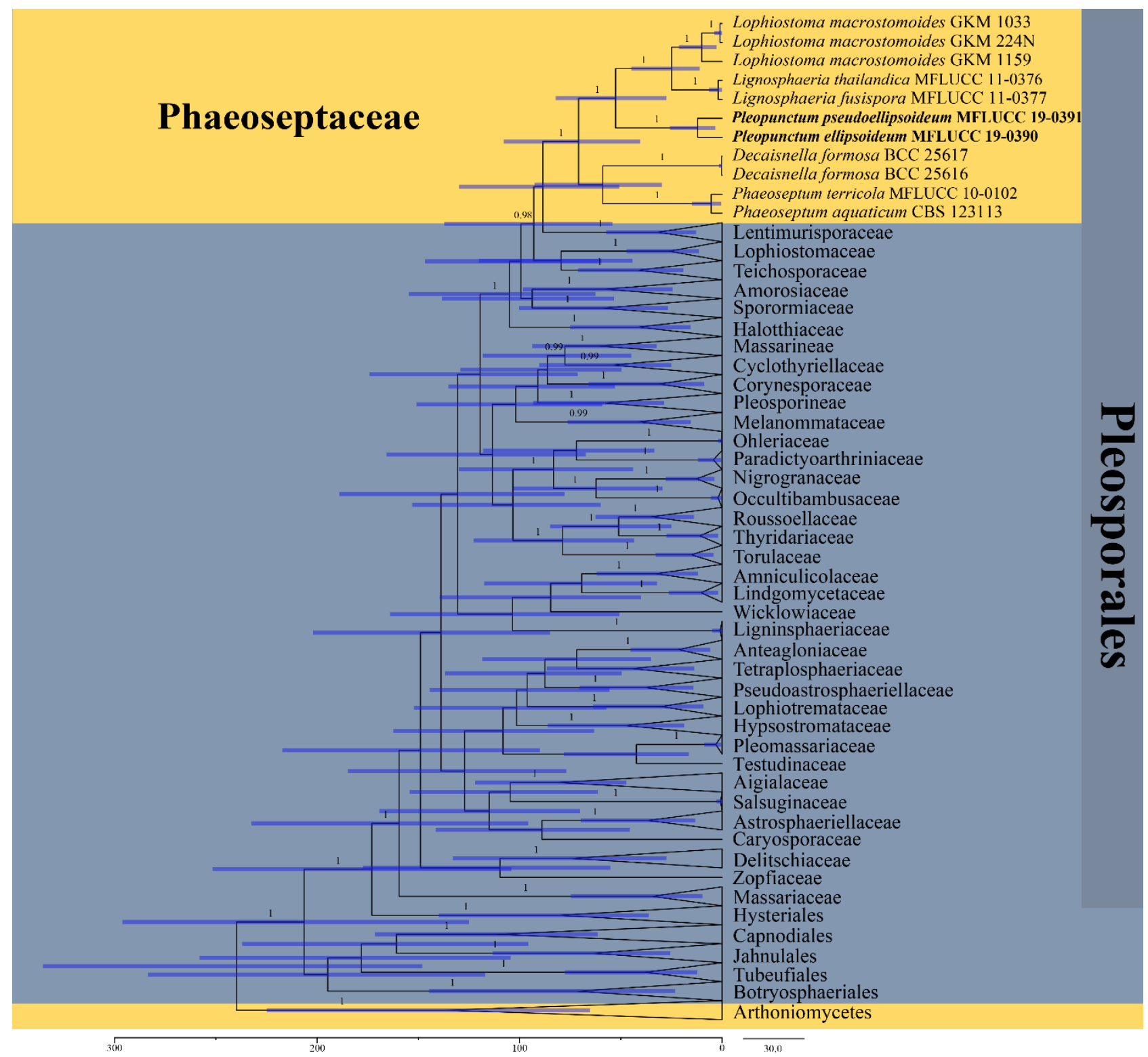

Figure 2 - Maximum clade credibility (MCC) tree with divergence times estimates obtained from BEAST. Posterior probabilities for branch support greater than 0.95 are given. Bars correspond to the $95 \%$ highest posterior density intervals. The new taxa are indicated in bold.

The most likely tree (-ln $=22570.586787)$ is presented (Fig. 1). The matrix had 1,462 distinct alignment patterns with $38.91 \%$ undetermined characters or gaps. The two Pleopunctum taxa clustered together with maximal support (ML-bs $=100 \%$, MP-bs $=100 \%$, PP $=1.00$ ) in Phaeoseptaceae. Pleopunctum subclade is close to Lignosphaeria and Thyridaria macrostomoides with maximal support (ML-bs $=100 \%$, MP-bs $=100 \%, \mathrm{PP}=1.00$ ) in Phaeoseptaceae.

Neolophiostoma pigmentatum Boonmee \& K.D. Hyde (strain MFLUCC 10-0129) was included in Phaeoseptaceae and formed a basal subclade in the phylogenetic analyses of combined LSU, SSU and TEF1 $\alpha$ sequence data carried out by Hyde et al. (2018). However, in our analysis, Neolophiostoma belongs to Halotthiaceae. The monophyly of Phaeoseptaceae is well-supported by ML and Bayesian analyses, while Halotthiaceae is only supported by Bayesian analysis. Moreover, Halotthiaceae and Phaeoseptaceae clades had a sister relationship, but the monophyly of these two clades is not supported by ML, MP and Bayesian analyses. Among the seven families, the monophyly of Amorosiaceae, Lentimurisporaceae, Lophiostomaceae, Phaeoseptaceae and Teichosporaceae are well-supported. 


\section{Divergence time estimations}

According to the divergence times estimates (Fig. 2), the crown age of Dothideomycetes (which comprised taxa in Botryosphaeriales, Capnodiales, Hysteriales, Jahnulales, Pleosporales and Tubeufiales) is around 206 MYA. The orders Hysteriales and Pleosporales diverged approximately 172 MYA. The crown age of Pleosporales is around 159 MYA. Among the Pleosporales families, Phaeoseptaceae diverged with Lentimurisporaceae approximately 88 MYA and the crown age of Phaeoseptaceae is around 71 MYA. Thus, the establishment of Phaeoseptaceae is supported and corresponds to previous studies (Liu et al. 2017, Zhang et al. 2019).

\section{Taxonomy}

Pleopunctum N.G. Liu, K.D. Hyde \& J.K. Liu, gen. nov. Index Fungorum: IF556522; Facesoffungi number: FoF 06113

Etymology - "Pleo-" an abbreviation of Pleosporales, the order in which this fungus is classified; "punctum" in reference to the punctiform colonies on natural substrate.

Saprobic on decaying wood in terrestrial habitats. Sexual morph: Undetermined. Asexual morph: Hyphomycetous. Colonies on natural substrate sporodochial, superficial, brown, scattered, gregarious, punctiform. Mycelium immersed in the substratum, composed of septate, branched, subhyaline to greyish brown hyphae. Conidiophores macronematous, mononematous, cylindrical, branched, septate, medium brown, smooth-walled, thick-walled. Conidiogenous cells monoblastic, cylindrical, brown. Conidia acrogenous, solitary, muriform, constricted at septa, broadly oval to ellipsoidal, smooth-walled, pale brown when immature, broadly obtuse at apex and dark brown, truncate at base and paler brown when mature, often with a hyaline, elliptical to globose basal cell.

Type species - Pleopunctum ellipticum

Notes - Pleopunctum is the first hyphomycetous genus in Phaeoseptaceae. It contains two species, namely P. ellipticum and P. pseudoellipticum, and they formed a distinct subclade in Phaeoseptaceae in the phylogenetic tree. The sexual morph of Pleopunctum is unknown, and the asexual morph has a unique morphology compared to those phylogenetically related species. Pleopunctum is characterized by its macronematous, mononematous conidiophores, monoblastic conidiogenous cells and muriform, oval to ellipsoidal conidia often with a hyaline, elliptical to globose basal cell. We hereby introduce the new genus based on the distinctiveness of morphology and multi-gene phylogeny.

Pleopunctum ellipsoideum N.G. Liu, K.D. Hyde \& J.K. Liu, sp. nov.

Fig. 3

Index Fungorum: IF556523; Facesoffungi number: FoF 06114

Etymology - in reference to the ellipsoidal conidia

Holotype - MFLU 19-0685

Saprobic on decaying wood. Sexual morph: Undetermined. Asexual morph: Hyphomycetous. Colonies on natural substrate sporodochial, superficial, brown, scattered, gregarious, punctiform. Mycelium immersed in the substratum, composed of septate, branched, subhyaline to greyish brown hyphae. Conidiophores $1.5-3.5 \mu \mathrm{m}$ wide $(\bar{x}=2.5 \mu \mathrm{m}, \mathrm{n}=15)$, macronematous, mononematous, cylindrical, branched, septate, medium brown, smooth-walled, thick-walled. Conidiogenous cells monoblastic, terminal, integrated, medium brown. Conidia 39-51 $\times 17-24 \mu \mathrm{m}(\bar{x}=45 \times 20 \mu \mathrm{m}, \mathrm{n}$ $=30$ ), acrogenous, solitary, muriform, constricted at septa, oval to ellipsoidal, smooth-walled, pale brown when immature, broadly obtuse at apex and dark brown, truncate at base and paler brown when mature, often with a hyaline, elliptical to globose basal cell, 8-20 × 8.5-18.5 $\mu \mathrm{m}(\bar{x}=13 \times 13$ $\mu \mathrm{m}, \mathrm{n}=30)$.

Culture characteristics - Conidium germinated on water agar within 24 hours. Germ tubes produced from basal cell. Mycelia superficial, irregular circular, grey in the central cycle and pale grey in the outer circle from above. Dark brown in the central cycle and yellowish brown in the outer circle from below.

Material examined - CHINA, Guizhou Province, Guiyang, Guizhou Academy of Agricultural 
Sciences, on decaying wood, 7 June 2018, N.G. Liu, NKY027 (MFLU 19-0685); ex-type living culture, MFLUCC 19-0390.

Pleopunctum pseudoellipsoideum N.G. Liu, K.D. Hyde \& J.K. Liu, sp. nov.

Fig. 4

Index Fungorum: IF556524; Facesoffungi number: FoF 06115

Etymology - in reference to its similar mophology with P. ellipticum.

Holotype - MFLU 19-0686

Saprobic on decaying wood. Sexual morph: Undetermined. Asexual morph: Hyphomycetous. Colonies on natural substrate sporodochial, superficial, black, scattered, gregarious, punctiform. Mycelium immersed in the substratum, composed of septate, branched hyphae. Conidiophores 1.5$4.5 \mu \mathrm{m}$ wide $(\bar{x}=3 \mu \mathrm{m}, \mathrm{n}=15)$, macronematous, mononematous, cylindrical, wider at the tip, septate, medium brown, smooth-walled, thick-walled. Conidiogenous cells monoblastic, terminal, integrated, medium brown. Conidia 39-59 $\times 19-28 \mu \mathrm{m}(\bar{x}=50 \times 24 \mu \mathrm{m}, \mathrm{n}=30)$, acrogenous, solitary, muriform, constricted at septa, oval to ellipsoidal, smooth-walled, broadly obtuse at apex and dark brown, truncate at base and paler brown, often with a hyaline, elliptical to subglobose basal cell, $6.5-13.5 \times 11-15.5 \mu \mathrm{m}(\bar{x}=11-13 \mu \mathrm{m}, \mathrm{n}=15)$.

Culture characteristics - Conidium germinated on water agar within 24 hours. Germ tubes produced from basal cell. Mycelia superficial, irregular circular, grey in the center and greyish white near the edge from above. Dark brown in the center, becoming paler towards the edge from below.

Material examined - China, Guizhou Province, Zunyi, Wangcao, on decaying wood, 16 September 2018, N.G. Liu, KKS020 (MFLU 19-0686); ex-type living culture, MFLUCC 19-0391.

Notes - Pleopunctum ellipsoideum and P. pseudoellipticum are morphologically similar. They both have sporodochial conidiomata, mononematous, cylindrical conidiophores, monoblastic, terminal conidiogenous cells and muriform, oval to ellipsoidal conidia often with a hyaline, elliptical to globose basal cell. The conidia size is also similar (39-51 × 17-24 $\mu \mathrm{m}$ vs. 39-59 × 19$28 \mu \mathrm{m})$. However, ITS comparison between P. ellipsoideum and P. pseudoellipsoideum showed that there are 12 bp differences (including the gaps) in a total of $524 \mathrm{bp}$, and $30 \mathrm{bp}$ differences in a total of 985 bp for TEFla (Table 3). Therefore, we identify $P$. ellipsoideum and $P$. pseudoellipsoideum as distinct species following the guidelines for species delineation in Jeewon \& Hyde (2016).

\section{Discussion}

In our studies, Pleopunctum ellipsoideum and P. pseudoellipsoideum were collected from the same province but different cities in China. They are both saprobic on unidentified decaying woods. Based on phylogenetic analyses of combined LSU, SSU, ITS and TEF1 $\alpha$ sequence data, $P$. ellipsoideum and $P$. pseudoellipsoideum formed a well-supported subclade and did not belong to any existing genera in Phaeoseptaceae. Moreover, although $P$. ellipsoideum and $P$. pseudoellipsoideum share similar morphology, they formed independent and distinct lineages with different branch lengths. Sufficient nucleotide differences are noted between P. ellipsoideum and $P$. pseudoellipsoideum in the ITS, LSU and TEF1 $\alpha$ regions (Table 3). Therefore, we propose Pleopunctum as a new genus in Phaeoseptaceae and identify $P$. ellipsoideum and $P$. pseudoellipsoideum as different species.

Hyde et al. (2018) established the family Phaeoseptaceae to accommodate the genera Lignosphaeria Boonmee et al., Neolophiostoma Boonmee \& K.D. Hyde and Phaeoseptum Ying Zhang, J. Fourn. \& K.D. Hyde, along with putatively named species Decaisnella formosa AbdelWahab \& E.B.G. Jones and Thyridaria macrostomoides (De Not.) M.E. Barr, based on LSU, SSU and TEF1 $\alpha$ sequence data. Phaeoseptaceae is characterized by subglobose to globose ascomata with short papilla, bitunicate, long pedicellate, 8-spored asci with a small ocular chamber, and light brown, multi-septate ascospores (Hyde et al. 2018). No hyphomycetous asexual morphs were reported in this family. Thus, morphological comparison between Pleopunctum and other genera in Phaeoseptaceae is not available. The monotypic genus Neolophiostoma was introduced by 
Ariyawansa et al. (2015) with the type species $N$. pigmentatum in the family Halotthiaceae. However, Neolophiostoma was transferred to Phaeoseptaceae by Hyde et al. (2018). In our analysis, Neolophiostoma remained within Halotthiaceae, although without good support, but agrees with Ariyawansa et al. (2015) based on LSU, SSU, RPB2 sequence data; Hyde et al. (2016) based on LSU and SSU sequence data and Phukhamsakda et al. (2016a) based on LSU, SSU, ITS, TEF1 $\alpha$ and RPB2 sequence data.

Neolophiostoma resembles Halotthia, the type genus of Halotthiaceae, in having ostiolate ascomata, bitunicate, 8-spored and fissitunicate asci with an ocular chamber, but they differ in that Neolophiostoma has hyaline, 3-5-septate ascospores with a thin gelatinous sheath while Halotthia has brown, 1-septate ascospores. Therefore, Neolophiostoma probably belongs to Halotthiaceae or has its own family, but more collections and analyses are warranted to verify any taxonomic assumption. Our divergence time estimation shows that Phaeoseptaceae diverged with Lentimurisporaceae approximately 88 MYA, the establishment of the Phaeoseptaceae agrees well with ages (50-150 MYA) proposed by Hyde et al. (2017), with the genus Neolophiostoma excluded. Our work generated similar results with Liu et al. (2017) and Zhang et al. (2019). Decaisnella was introduced by Fabre (1879) based on D. spectabilis Fabre, and there are fourteen epithets listed in Index Fungorum (May 2019). However, sequence data are only available for $D$. formosa. Because of the lack of molecular sequence data of $D$. spectabilis, the phylogenetic placement of the genus Decaisnella requires further confirmation. Thyridaria macrostomoides was described by Barr (1990), until twenty year later, Mugambi \& Huhndorf (2009) provided the DNA sequence data of $T$. macrostomoides from three newly obtained collections (GKM 1033, GKM 1159, GKM 224N) and placed them in Lophiostomaceae. Thambugala et al. (2015) restudied Lophiostomaceae and they excluded these three T. macrostomoides strains from Lophiostomaceae and assigned them as Dothideomycetes genera incertae sedis. However, Thyridaria Sacc. was accommodated to Thyridariaceae based on the type species T. broussonetiae (Sacc.) Traverso (Hyde et al. 2013, Jaklitsch \& Voglmayr 2016). Therefore, these three T. macrostomoides collections may have been wrongly identified because they are distant from $T$. broussonetiae, and they probably can be recognized as a new genus, but we are not willing to introduce it in this study until we examine the type materials of T. macrostomoides.

Table 3 Nucleotide differences in the ITS, LSU and TEF1 $\alpha$ regions for P. ellipsoideum and P. pseudoellipsoideum. Numbers are in reference to the nucleotide position of DNA sequences (P. ellipsoideum) submitted in GenBank.

\begin{tabular}{|c|c|c|c|c|c|c|c|c|c|c|c|c|c|c|c|c|c|c|c|c|c|c|c|}
\hline \multirow{2}{*}{ Species } & \multicolumn{12}{|l|}{ ITS } & \multicolumn{3}{|c|}{ LSU } & \multicolumn{8}{|c|}{ TEF1a } \\
\hline & 12 & 15 & 45 & 91 & 143 & 144 & 145 & 338 & 339 & 359 & 462 & 525 & 85 & 101 & 160 & 8 & 12 & 33 & 42 & 150 & 153 & 171 & 186 \\
\hline P. ellipsoideum & $\mathrm{C}$ & $G$ & $\mathrm{~T}$ & $G$ & $\mathrm{~A}$ & $\mathrm{~A}$ & $\mathrm{~A}$ & $\mathrm{~T}$ & $\mathrm{C}$ & $\mathrm{C}$ & $\mathrm{A}$ & - & $\mathrm{C}$ & $G$ & $\mathrm{C}$ & $\mathrm{T}$ & $\mathrm{T}$ & $\mathrm{T}$ & $\mathrm{T}$ & $\mathrm{T}$ & $\mathrm{T}$ & $G$ & $\mathrm{~T}$ \\
\hline P. pseudoellipsoideum & $\mathrm{T}$ & $\mathrm{C}$ & - & A & - & - & - & $\mathrm{C}$ & $\mathrm{T}$ & $\mathrm{T}$ & - & $\mathrm{T}$ & $\mathrm{T}$ & A & $\mathrm{T}$ & $\mathrm{C}$ & $\mathrm{C}$ & $\mathrm{C}$ & $\mathrm{C}$ & $\mathrm{C}$ & $\mathrm{C}$ & $\mathrm{T}$ & $\mathrm{C}$ \\
\hline \multirow{2}{*}{ Species } & \multicolumn{23}{|c|}{ TEF1a } \\
\hline & 252 & 258 & 343 & 366 & 397 & 571 & 572 & 591 & 684 & 705 & 876 & 909 & 915 & 924 & 936 & 951 & 965 & 969 & 975 & 979 & 984 & 986 & \\
\hline P. ellipsoideum & $\mathrm{C}$ & $\mathrm{C}$ & $\mathrm{T}$ & $\mathrm{C}$ & $\mathrm{T}$ & $\mathrm{A}$ & $\mathrm{C}$ & $\mathrm{T}$ & $\mathrm{T}$ & $\mathrm{C}$ & $\mathrm{C}$ & $\mathrm{C}$ & $\mathrm{C}$ & G & $\mathrm{T}$ & $\mathrm{C}$ & $G$ & $\mathrm{~T}$ & $\mathrm{C}$ & $\mathrm{G}$ & $\mathrm{A}$ & $\mathrm{A}$ & \\
\hline P. pseudoellipsoideum & $\mathrm{T}$ & $\mathrm{T}$ & A & $\mathrm{T}$ & A & $\mathrm{G}$ & $\mathrm{T}$ & $\mathrm{C}$ & $\mathrm{C}$ & $\mathrm{T}$ & $\mathrm{T}$ & $\mathrm{T}$ & $\mathrm{T}$ & $\mathrm{T}$ & $\mathrm{C}$ & $\mathrm{T}$ & $\mathrm{T}$ & $\mathrm{C}$ & $\mathrm{T}$ & $\mathrm{C}$ & $\mathrm{T}$ & $\mathrm{C}$ & \\
\hline
\end{tabular}



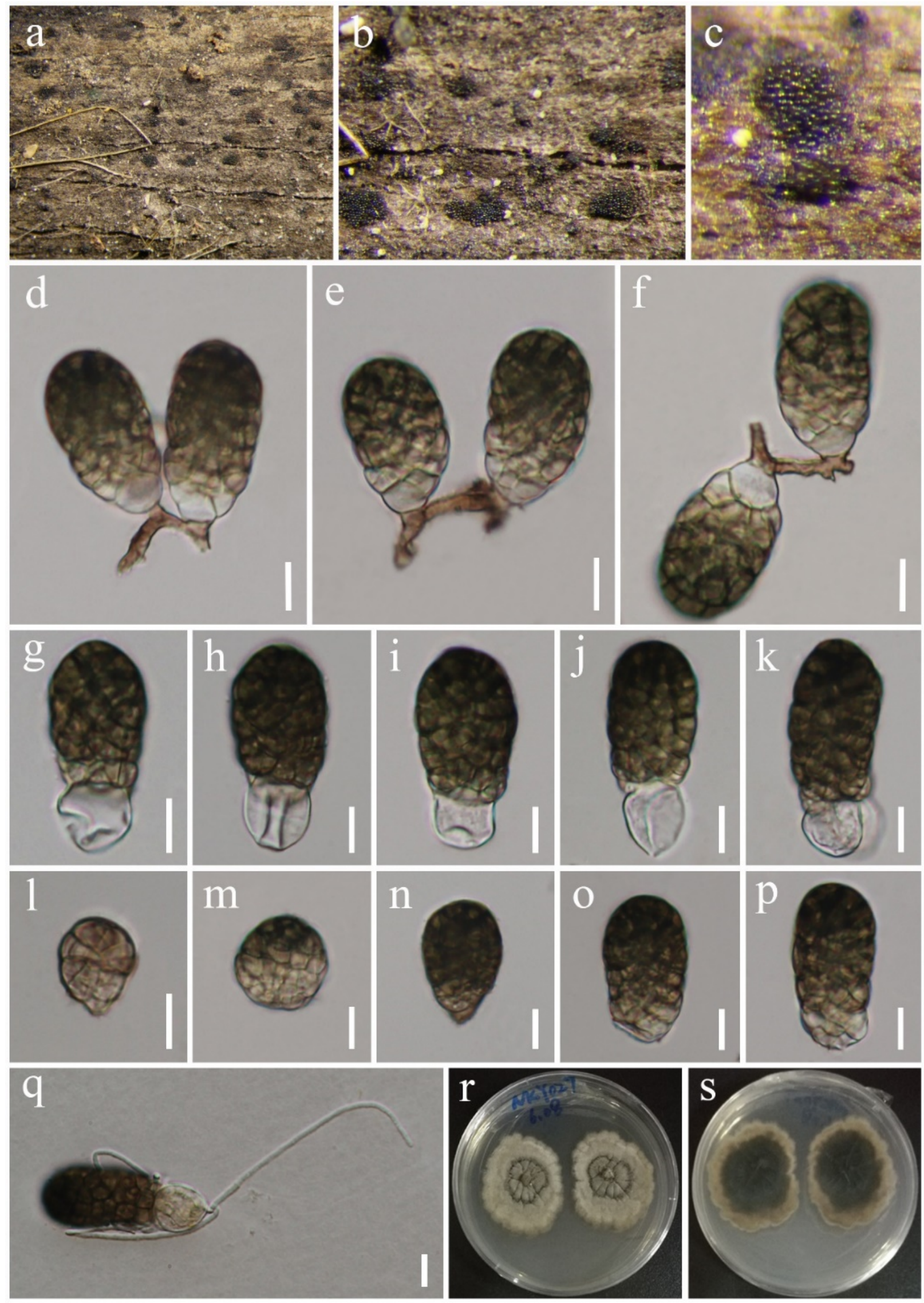

Figure 3 - Pleopunctum ellipsoideum (MFLU 19-0685, holotype). a-c Colonies on natural substrates. $\mathrm{d}-\mathrm{f}$ Conidiophores and conidia. g-k Conidia with basal hyaline cells. $\mathrm{l}-\mathrm{p}$ Conidia without basal hyaline cells. q Germinated conidium. r, s Colonies on PDA media. Scale bars: $\mathrm{d}-\mathrm{q}=$ $10 \mu \mathrm{m}$ 

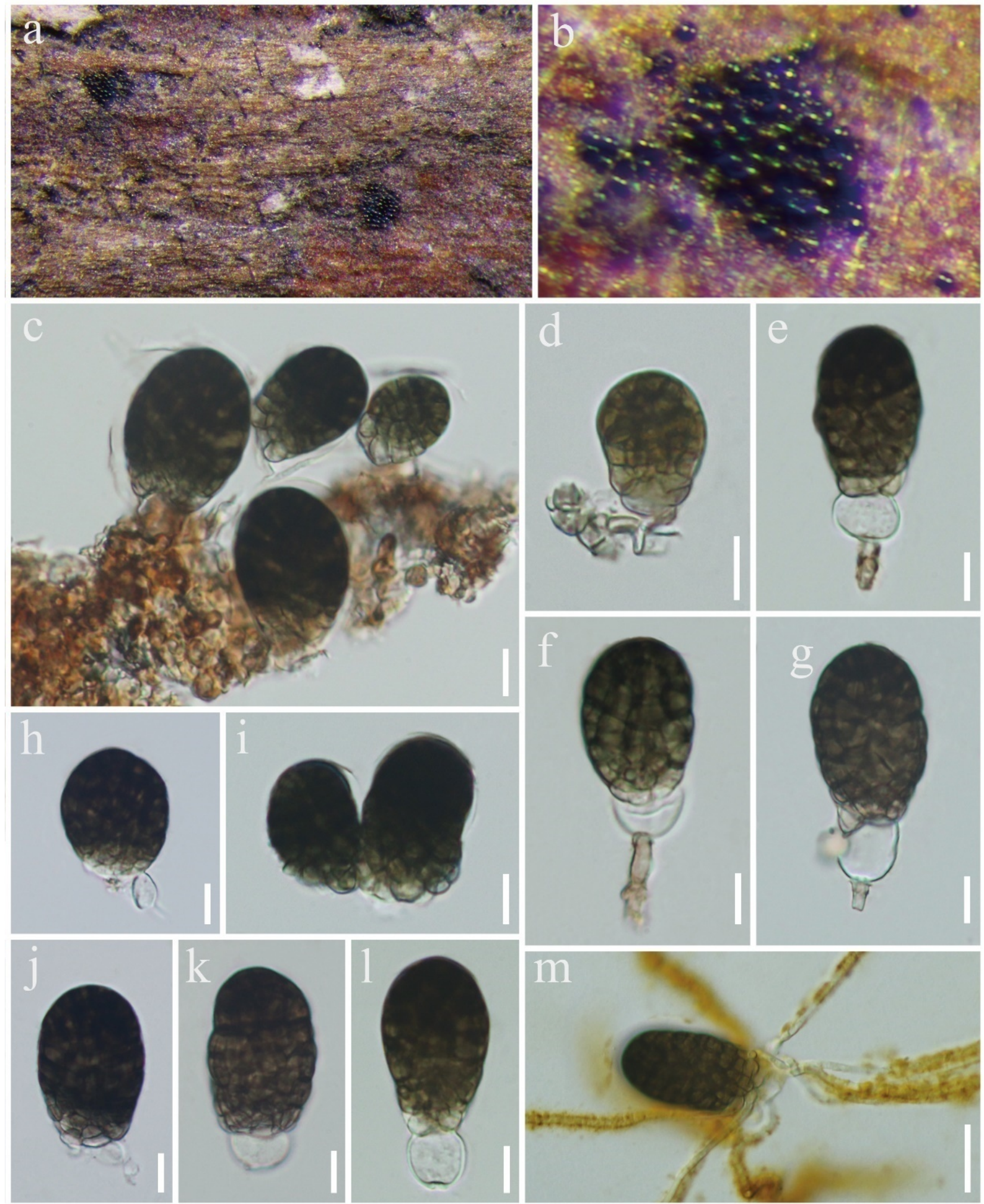

Figure 4 - Pleopunctum pseudoellipsoideum (MFLU 19-0686, holotype). a-b Colonies on natural substrates. c Conidia on substrate. d-g Conidiophores and conidia. h-l Conidia with or without basal hyaline cells. $\mathrm{m}$ Germinated conidium. Scale bars: $\mathrm{c}-\mathrm{l}=10 \mu \mathrm{m}, \mathrm{m}=20 \mu \mathrm{m}$

Among the six phylogenetically close families in this study, only coelomycetous asexual morphs have been reported in Halotthiaceae, Lophiostomaceae and Teichosporaceae (Hyde et al. 2013, Thambugala et al. 2015). Amorosiaceae includes two genera, Amorosia Mantle \& D. Hawksw. and Angustimassarina Thambugala, Kaz. Tanaka \& K.D. Hyde, along two putative strains, Exosporium stylobatum CBS 160.30 and Massarina corticola CBS 154.93 (Thambugala et 
al. 2015). Amorosia and Angustimassarina can produce chlamydospores in cultures, which are characterized by micronematous to semi-macronematous conidiophores, elongate-clavate conidia. However, Amorosia has 3-4-septate conidia with a distinct central pore in each septum, while Angustimassarina has 1-3-septate conidia (Mantle et al. 2006, Thambugala et al. 2015). Exosporium is a hyphomycetous genus with loosely aggregated conidiophores, terminal or lateral conidiogenous cells with prominent loci, and 5-distoseptate conidia (Crous et al. 2011). Lentimurisporaceae is a dematiaceous hyphomycetous family without known sexual morphs. The type genus Lentimurispora produces muriform, lenticular conidia with dark brown central cells and subhyaline to pale brown peripheral cells (Liu et al. 2018). Bahusandhika Subram. is a torula-like genus with fusiform, cylindrical or rhomboidal conidia formed simply or in branched chains. Berkleasmium Zobel has been shown to be not monophyletic (Pinnoi et al. 2007). Conidial morphology of Berkleasmium is oval to ellipsoidal, often with a protruding hilum instead of a hyaline, elliptical to globose basal cell (Ellis 1971, Bussaban et al. 2001). The genus Sparticola Phukhams., Ariyaw., Camporesi \& K.D. Hyde in Sporormiaceae can form hyphomycetous asexual morph in culture which is characterized by semi-macronematous to macronematous, branched conidiophores, annellidic, doliform conidiogenous cells, and dictyosporous conidia with granules. Other genera in Sporormiaceae, such as Forliomyces Phukhams., Camporesi \& K.D. Hyde, produce coelomycetous asexual morphs. Therefore, Pleopunctum has a unique asexual morph morphology in the family Phaeoseptaceae, as well as compared to its phylogenetically close families in Pleosporales.

\section{Acknowledgements}

This work was funded by the Joint Fund of the National Natural Science Foundation of China and the Karst Science Research Center of Guizhou province (Grant No. U1812401). The Faculty of Agriculture, Natural Resources and Environment, Naresuan University is acknowledged for providing Ph.D. research scholarship to Ning-Guo Liu. Kevin D. Hyde would thank the Thailand Research grants entitled "Impact of climate change on fungal diversity and biogeography in the Greater Mekong Subregion” (grant no: RDG6130001). We are grateful to Dr. Shaun Pennycook for providing advice on fungal nomenclature.

\section{References}

Ariyawansa HA, Hyde KD, Jayasiri SC, Buyck B et al. 2015 - Fungal diversity notes 111-252 taxonomic and phylogenetic contributions to fungal taxa. Fungal Diversity 75, 27-274.

Barr ME. 1990 - Melanommatales (Loculoascomycetes). North American Flora 13, 1-129.

Bhat DJ. 2010 - Fascinating microfungi (Hyphomycetes) of Western Ghats-India. Broadway Book Centre.

Bhat DJ, Kendrick B. 1993 - Twenty-five new conidial fungi from the Western Ghats and the Andaman Islands (India). Mycotaxon 49, 19-90.

Boonmee S, D’souza MJ, Luo ZL, Pinruan U et al. 2016 - Dictyosporiaceae fam. nov. Fungal Diversity 80, 457-482.

Bussaban B, Lumyong S, Lumyong P, McKenzie EHC, Hyde KD. 2001 - A synopsis of the genus Berkleasmium with two new species and new records of Canalisporium caribense from Zingiberaceae in Thailand. Fungal Diversity 8, 73-85.

Chomnunti P, Hongsanan S, Aguirre-Hudson B, Tian Q et al. 2014 - The sooty moulds. Fungal Diversity 66, 1-36.

Crous PW, Summerell BA, Shivas RG, Romberg M et al. 2011 - Fungal Planet description sheets: 92-106. Persoonia 27, 130-162.

Dai DQ, Phookamsak R, Wijayawardene NN, Li WJ et al. 2017 - Bambusicolous fungi. Fungal Diversity 82, 1-105.

Doilom M, Dissanayake AJ, Wanasinghe DN, Boonmee S et al. 2017 - Microfungi on Tectona grandis (teak) in Northern Thailand. Fungal Diversity 82, 107-182. 
Dong W, Hyde KD, Bhat DJ, Zhang H. 2018 - Introducing Aculeata aquatica gen. et sp. nov., Minimelanolocus thailandensis sp. nov. and Thysanorea aquatica sp. nov. (Herpotrichiellaceae, Chaetothyriales) from freshwater in northern Thailand. Mycological Progress 17, 617-629.

Drummond AJ, Suchard MA, Xie D, Rambaut A. 2012 - Bayesian phylogenetics with BEAUti and the BEAST 1.7. Molecular Biology and Evolution 29, 1969-1973.

Ellis MB. 1971 - Dematiaceous hyphomycetes. Commonwealth Mycological Institute, Kew, Surrey, England.

Ellis MB. 1976 - More Dematiaceous Hyphomycetes Commonwealth Mycological Institute, Kew, Surrey, England.

Fabre JH. 1879 - Essai sur les Sphériacées du Départment de Vaucluse. Annales des Sciences Naturelles Botanique ser. 6. 9, 66-118.

Goh TK, Ho WH, Hyde KD, Whitton SR, Umali TE. 1998 - New records and species of Canalisporium (Hyphomycetes), with a revision of the genus. Canadian Journal of Botany 76, 142-152.

Hall TA. 1999 - BioEdit: a user-friendly biological sequence alignment editor and analysis program for Windows 95/98/NT. In: Nucleic Acids Symposium Series, pp 95-98.

Hashimoto A, Matsumura M, Hirayama K, Tanaka K. 2017 - Revision of Lophiotremataceae sensu lato (Pleosporales, Dothideomycetes): establishment of Aquasubmersaceae, Cryptocoryneaceae, Hermatomycetaceae fam. nov. Persoonia 39, 51-73.

Hillis DM, Bull JJ. 1993 - An empirical test of bootstrapping as a method for assessing confidence in phylogenetic analysis. Systematic Biology 42, 182-192.

Hongsanan S, Sánchez-Ramírez S, Crous PW, Ariyawansa HA et al. 2016 - The evolution of fungal epiphytes. Mycosphere 7, 1690-1712.

Hu DM, Cai L, Chen H, Bahkali AH, Hyde KD. 2010 - Four new freshwater fungi associated with submerged wood from Southwest Asia. Sydowia 62, 191-203.

Hyde KD, Chaiwan N, Norphanphoun C, Boonmee S et al. 2018 - Mycosphere notes 169-224. Mycosphere 9, 271-430.

Hyde KD, Hongsanan S, Jeewon R, Bhat DJ et al. 2016 - Fungal diversity notes 367-490: taxonomic and phylogenetic contributions to fungal taxa. Fungal Diversity 80, 1-270.

Hyde KD, Jones EBG, Liu JK, Ariyawansa HA et al. 2013 - Families of Dothideomycetes. Fungal Diversity 63, 1-313.

Hyde KD, Maharachchikumbura SSN, Hongsanan S, Samarakoon MC et al. 2017 - The ranking of fungi: a tribute to David L. Hawksworth on his 70th birthday. Fungal Diversity 84, 1-23.

Jaklitsch WM, Voglmayr H. 2016 - Hidden diversity in Thyridaria and a new circumscription of the Thyridariaceae. Studies in Mycology 85, 35-64.

Jayasiri SC, Hyde KD, Ariyawansa HA, Bhat J et al. 2015 - The Faces of Fungi database: fungal names linked with morphology, phylogeny and human impacts. Fungal Diversity 74, 3-18.

Jeewon R, Hyde KD. 2016 - Establishing species boundaries and new taxa among fungi: recommendations to resolve taxonomic ambiguities. Mycosphere 7, 1669-1677.

Jeewon R, Liew ECY, Hyde KD. 2003 - Molecular systematics of the Amphisphaeriaceae based on cladistic analyses of partial LSU rDNA gene sequences. Mycological Research 107, 1392-1402.

Katoh K, Standley DM. 2013 - MAFFT multiple sequence alignment software version 7: improvements in performance and usability. Molecular Biology and Evolution 30, 772-780.

Klaubauf S, Tharreau D, Fournier E, Groenewald JZ et al. 2014 - Resolving the polyphyletic nature of Pyricularia (Pyriculariaceae). Studies in Mycology 79, 85-120.

Kruys Å, Wedin M. 2009 - Phylogenetic relationships and an assessment of traditionally used taxonomic characters in the Sporormiaceae (Pleosporales, Dothideomycetes, Ascomycota), utilising multi-gene phylogenies. Systematics and Biodiversity 7, 465-478.

Larsson A. 2014 - AliView: a fast and lightweight alignment viewer and editor for large datasets. Bioinformatics 30, 3276-3278. 
Leão-Ferreira SM, Pascholati Gusmão LF, Castañeda Ruiz RF. 2013 - Conidial fungi from the semi-arid Caatinga biome of Brazil. Three new species and new records. Nova Hedwigia 96, 479-494.

Liu JK, Chomnunti P, Cai L, Phookamsak R et al. 2010 - Phylogeny and morphology of Neodeightonia palmicola sp. nov. from palms. Sydowia 62, 261-276.

Liu JK, Hyde KD, Jeewon R, Phillips AJL et al. 2017 - Ranking higher taxa using divergence times: a case study in Dothideomycetes. Fungal Diversity 84, 75-99.

Liu NG, Lin CG, Liu JK, Samarakoon MC et al. 2018 - Lentimurisporaceae, a new pleosporalean family with divergence times estimates. Cryptogamie, Mycologie 39, 259-282.

Lu YZ, Liu JK, Hyde KD, Jeewon R et al. 2018 - A taxonomic reassessment of Tubeufiales based on multi-locus phylogeny and morphology. Fungal Diversity 92, 131-344.

Luo ZL, Bhat DJ, Jeewon R, Boonmee S et al. 2017 - Molecular phylogeny and morphological characterization of asexual fungi (Tubeufiaceae) from freshwater habitats in Yunnan, China. Cryptogamie, Mycologie 38, 27-53.

Maharachchikumbura SSN, Hyde KD, Jones EBG, McKenzie EHC et al. 2016 - Families of Sordariomycetes. Fungal Diversity 79, 1-317.

Mantle PG, Hawksworth DL, Pazoutova S, Collinson LM, Rassing BR. 2006 - Amorosia littoralis gen. sp. nov., a new genus and species name for the scorpinone and caffeine-producing hyphomycete from the littoral zone in the Bahamas. Mycological Research 110, 1371-1378.

Mindell RA, Stockey RA, Beard G, Currah RS. 2007 - Margaretbarromyces dictyosporus gen. sp. nov.: a permineralized corticolous ascomycete from the Eocene of Vancouver Island, British Columbia. Mycological Research 111, 680-684.

Mugambi GK, Huhndorf SM. 2009 - Molecular phylogenetics of Pleosporales: Melanommataceae and Lophiostomataceae re-circumscribed (Pleosporomycetidae, Dothideomycetes, Ascomycota). Studies in Mycology 64, 103-121.

Nylander JA. 2004 - MrModeltest v2 , Program distributed by the author, Evolutionary Biology Centre, Uppsala University, Uppsala, Sweden.

Pérez-Ortega S, Garrido-Benavent I, Grube M, Olmo R, de los Ríos A. 2016 - Hidden diversity of marine borderline lichens and a new order of fungi: Collemopsidiales (Dothideomyceta). Fungal Diversity 80, 285-300.

Phukhamsakda C, Ariyawansa HA, Phillips AJL, Wanasinghe DN et al. 2016a - Additions to Sporormiaceae: Introducing two novel genera, Sparticola and Forliomyces, from Spartium. Cryptogamie, Mycologie 37, 75-97.

Phukhamsakda C, Hongsanan S, Ryberg M, Ariyawansa HA et al. 2016b - The evolution of Massarineae with Longipedicellataceae fam. nov. Mycosphere 7, 1713-1731.

Pinnoi A, Jeewon R, Sakayaroj J, Hyde KD, Jones EBG. 2007 - Berkleasmium crunisia sp. nov. and its phylogenetic affinities to the Pleosporales based on 18S and 28S rDNA sequence analyses. Mycologia 99, 378-384.

Rambaut A. 2006 - FigTree. Tree figure drawing tool version 1.3.1, Institute of Evolutionary Biology, University of Edinburgh.

Rambaut A, Suchard M, Xie D, Drummond A. 2014 - Tracer v1. 6. Available from: http://tree.bio.ed.ac.uk/software/tracer/

Rannala B, Yang ZH. 1996 - Probability distribution of molecular evolutionary trees: a new method of phylogenetic inference. Journal of Molecular Evolution 43, 304-311.

Rehner SA, Buckley E. 2005 - A Beauveria phylogeny inferred from nuclear ITS and EF1- $\alpha$ sequences: evidence for cryptic diversification and links to Cordyceps teleomorphs. Mycologia 97, 84-98.

Rikkinen J, Dörfelt H, Schmidt AR, Wunderlich J. 2003 - Sooty moulds from European Tertiary amber, with notes on the systematic position of Rosaria ('Cyanobacteria'). Mycological Research 107, 251-256.

Ronquist F, Teslenko M, van der Mark P, Ayres DL et al. 2012 - MrBayes 3.2: efficient Bayesian phylogenetic inference and model choice across a large model space. Systematic Biology 61, 
539-542.

Samarakoon MC, Hyde KD, Hongsanan S, McKenzie EHC et al. 2019 - Divergence time calibrations for ancient lineages of Ascomycota classification based on a modern review of estimations. Fungal Diversity 96, 285-346.

Seifert K, Morgan-Jones G, Gams W, Kendrick B. 2011 - The genera of Hyphomycetes. CBSKNAW Fungal Biodiversity Centre Utrecht.

Shenoy BD, Jeewon R, Hyde KD. 2007 - Impact of DNA sequence-data on the taxonomy of anamorphic fungi. Fungal Diversity 26, 1-54.

Shenoy BD, Jeewon R, Wu WP, Bhat DJ, Hyde KD. 2006 - Ribosomal and RPB2 DNA sequence analyses suggest that Sporidesmium and morphologically similar genera are polyphyletic. Mycological Research 110, 916-928.

Sri-Indrasutdhi V, Boonyuen N, Suetrong S, Chuaseeharonnachai C et al. 2010 - Wood-inhabiting freshwater fungi from Thailand: Ascothailandia grenadoidia gen. et sp. nov., Canalisporium grenadoidia sp. nov. with a key to Canalisporium species (Sordariomycetes, Ascomycota). Mycoscience 51, 411-420.

Stamatakis A. 2006 - RAxML-VI-HPC: maximum likelihood-based phylogenetic analyses with thousands of taxa and mixed models. Bioinformatics 22, 2688-2690.

Swofford DL. 2002 - PAUP*: phylogenetic analysis using parsimony (and other methods), version 4.0 b10. Sunderland, Massachusetts: Sinauer Associates Google Scholar.

Taylor JE, Hyde KD. 2003 - Microfungi of tropical and temperate palms. Fungal Diversity Research Series 12, 1-459.

Thambugala KM, Hyde KD, Tanaka K, Tian Q et al. 2015 - Towards a natural classification and backbone tree for Lophiostomataceae, Floricolaceae, and Amorosiaceae fam. nov. Fungal Diversity 74, 199-266.

Tsui CKM, Berbee ML, Jeewon R, Hyde KD. 2006 - Molecular phylogeny of Dictyosporium and allied genera inferred from ribosomal DNA. Fungal Diversity 21, 157-166.

Tsui CKM, Goh TK, Hyde KD. 2003 - Reflections on the genus Vanakripa, and a description of $V$. ellipsoidea sp. nov. Mycologia 95, 124-127.

Vilgalys R, Hester M. 1990 - Rapid genetic identification and mapping of enzymatically amplified ribosomal DNA from several Cryptococcus species. Journal of Bacteriology 172, 4238-4246.

Wang HK, Aproot A, Crous PW, Hyde KD, Jeewon R. 2007 - The polyphyletic nature of Pleosporales: an example from Massariosphaeria based on rDNA and RBP2 gene phylogenies. Fungal Biology 111, 1268-1276.

White TJ, Bruns T, Lee SJWT, Taylor JW. 1990 - Amplification and direct sequencing of fungal ribosomal RNA genes for phylogenetics. In: Innis MA, Gelfand DH, Sninsky JJ, White TJ, eds. PCR protocols: a guide to methods and applications. California, Academic Press. pp. 315- 322.

Wijayawardene NN, Hyde KD, Rajeshkumar KC, Hawksworth DL et al. 2017 - Notes for genera: Ascomycota. Fungal Diversity 86, 1-594.

Yang J, Liu JK, Hyde KD, Jones EBG, Liu ZY. 2018 - New species in Dictyosporium, new combinations in Dictyocheirospora and an updated backbone tree for Dictyosporiaceae. MycoKeys 36, 83-105.

Yang J, Maharachchikumbura SSN, Bhat DJ, Hyde KD et al. 2016 - Fuscosporellales, a new order of aquatic and terrestrial Hypocreomycetidae (Sordariomycetes). Cryptogamie, Mycologie 37, 449-475.

Zhang SN, Hyde KD, Jones EBG, Jeewon R et al. 2019 - Striatiguttulaceae, a new pleosporalean family to accommodate Longicorpus and Striatiguttula gen. nov. from palms. MycoKeys 49, 99-129.

Zhang Y, Fournier J, Phookamsak R, Bahkali AH, Hyde KD. 2013 - Halotthiaceae fam. nov. (Pleosporales) accommodates the new genus Phaeoseptum and several other aquatic genera. Mycologia 105, 603-609. 